\title{
Investigation of an Adenovirus-Induced Respiratory Disease Outbreak
}

\author{
Xingyi Geng, Ji Zhang, Guoliang Yang \\ Jinan Municipal Center for Disease Control and Prevention, Jinan, China. \\ Email: gengxingyi@163.com \\ Received September $10^{\text {th }}, 2013$; revised October $9^{\text {th }}, 2013$; accepted October $16^{\text {th }}, 2013$ \\ Copyright (C) 2013 Xingyi Geng, et al. This is an open access article distributed under the Creative Commons Attribution License, \\ which permits unrestricted use, distribution, and reproduction in any medium, provided the original work is properly cited.
}

\begin{abstract}
Objective: An epidemiological investigation was carried out in school X in Jinan, Shandong Province, China, to identify the cause, epidemiology, and etiological characteristics of a febrile respiratory disease outbreak; and therefore to control the dissemination. Methods: Both field epidemiological investigations and laboratory examinations were carried out. Results: Forty cases were identified, in which 38 cases were students and two were teachers. Clinical manifestations included fever, coughing, headache, and sore throat. A total of 21 pharyngeal swab specimens were collected and 18 tested positive for adenovirus. The adenovirus hexon gene was sequenced in three of the 18 positive specimens and the results showed a 100\% homology with the standard HAdV-55 HEXO. Conclusions: The outbreak originated from an adenovirus-infected student, who spread the pathogen to her classmates and teacher. The teacher then further disseminated the disease within the school which led to 40 febrile respiratory infections.
\end{abstract}

Keywords: Epidemiology; Research Methods; Laboratory Examination; Adenovirus; Respiratory Disease

\section{Background}

Adenoviruses (AdV) are DNA viruses that typically cause mild infections involving the upper or lower respiratory tract, gastrointestinal tract, or conjunctiva [1]. Replacement of dominant serotypes by new strains usually results in outbreaks in crowds. Outbreaks of AdV infection are more common in young children and military recruits [2]. So far, more than 65 serotypes (subdivided into groups $\mathrm{A}$ to $\mathrm{F}$ ) of $\mathrm{AdV}$ have been identified by DNA sequencing [3]. Of these, Ad-B55 was first isolated from an acute respiratory disease outbreak in Shaanxi Province, China in 2006. It is a re-emergent respiratory pathogen, which was shown to be an intraspecies recombination between HAdV-11a and HAdV-14 [4,5]. Since then, there have been no reported outbreaks worldwide.

In the present paper, we report an outbreak that involved 38 students and two teachers at school X in Jinan, Shandong Province, China. After an epidemiological investigation and DNA sequence homology, this outbreak was shown to be caused by Ad-B55. As far as we know, this is the second report on an outbreak of Ad-B55 in schools in China.

*Competing interests: The authors declare that they have no competing interests.

\section{Materials and Methods}

\subsection{Patients}

Within a week, 40 teachers and students from school X experienced at least one of following signs or symptoms: unexplained fever (with a body temperature $\geq 38^{\circ} \mathrm{C}$ ), coughing, sore throat, headache, throat congestion, swollen tonsils, purulent tonsils, or conjunctival hyperemia.

\subsection{Epidemiological Investigation}

An epidemiological investigation was carried out on the 40 reported cases to explore the possible cause and to control the dissemination. Data including demographic information, epidemiological characteristics, and clinical manifestations were collected and studied for each case. Throat swab specimens were sampled for some of the cases and sent for laboratory examinations.

\subsection{Laboratory Examinations}

Throat swab specimens from 21 cases and serum specimens from six acute cases were collected. Viruses were isolated and cultured using two types of cell lines: Hep 2 and Vero cells. Nucleic acid was extracted using the Ro- 
che high-purity viral nucleic acid kit (Roche Applied Science, Mannheim, Germany). Seven common respiratory tract pathogens were tested on 12 specimens using RT-PCR (or PCR), including influenza virus (A\B) respiratory syncytial virus, parainfluenza virus, rhinovirus, coronavirus $\mathrm{AdV}$, and bocavirus. All 21 specimens were tested for AdV using PCR. The sequencing results were analysed using DNAStar and Seqman. The nucleotide acid sequence homology was sequenced using MegAlign. Mega5 was used for sequence alignment and phylogenetic tree construction.

\subsection{Data Management and Statistics Analysis}

Epi Data 3.0 was used to establish a database and SPSS 17.0 software was used for statistical analysis.

\section{Results}

\subsection{School Background}

The incident school is located in the northwest of Jinan City, and covers an area of $14,700 \mathrm{~m}^{2}$ with a building area of $4,025 \mathrm{~m}^{2}$. The teaching block has four floors: grades 1 - 5 are located on levels one and two; grades 6 -
9 are located on levels three and four. There are two stairs in the building; and each grade is relatively independent. Teachers of each grade have separated offices. The highest incidence of this outbreak was from class 1 and 2 of grade 8, which are located in the east wing of the building (Figure 1).

There are 635 students (462 from grades $1-6,173$ from grades 7 - 9) and 72 members of staff in the school. Students are distributed in 17 classes from 9 grades. It is a non-boarding school with most of the students living in nearby villages.

Some of the students from grades 7 - 9 participated in the opening and closing ceremonies of a district sports competition on March $27^{\text {th }}$ and $28^{\text {th }} 2012$. It was the only social gathering of students from the school close to the time of the outbreak.

\subsection{Clinical Manifestations and Epidemiological Characteristics}

\subsubsection{Major Clinical Manifestations}

The main symptoms of the 40 reported cases included fever $\left(82.14 \%\right.$, highest reported body temperature $\left.40^{\circ} \mathrm{C}\right)$, coughing and sore throat $(78.57 \%)$, and headache

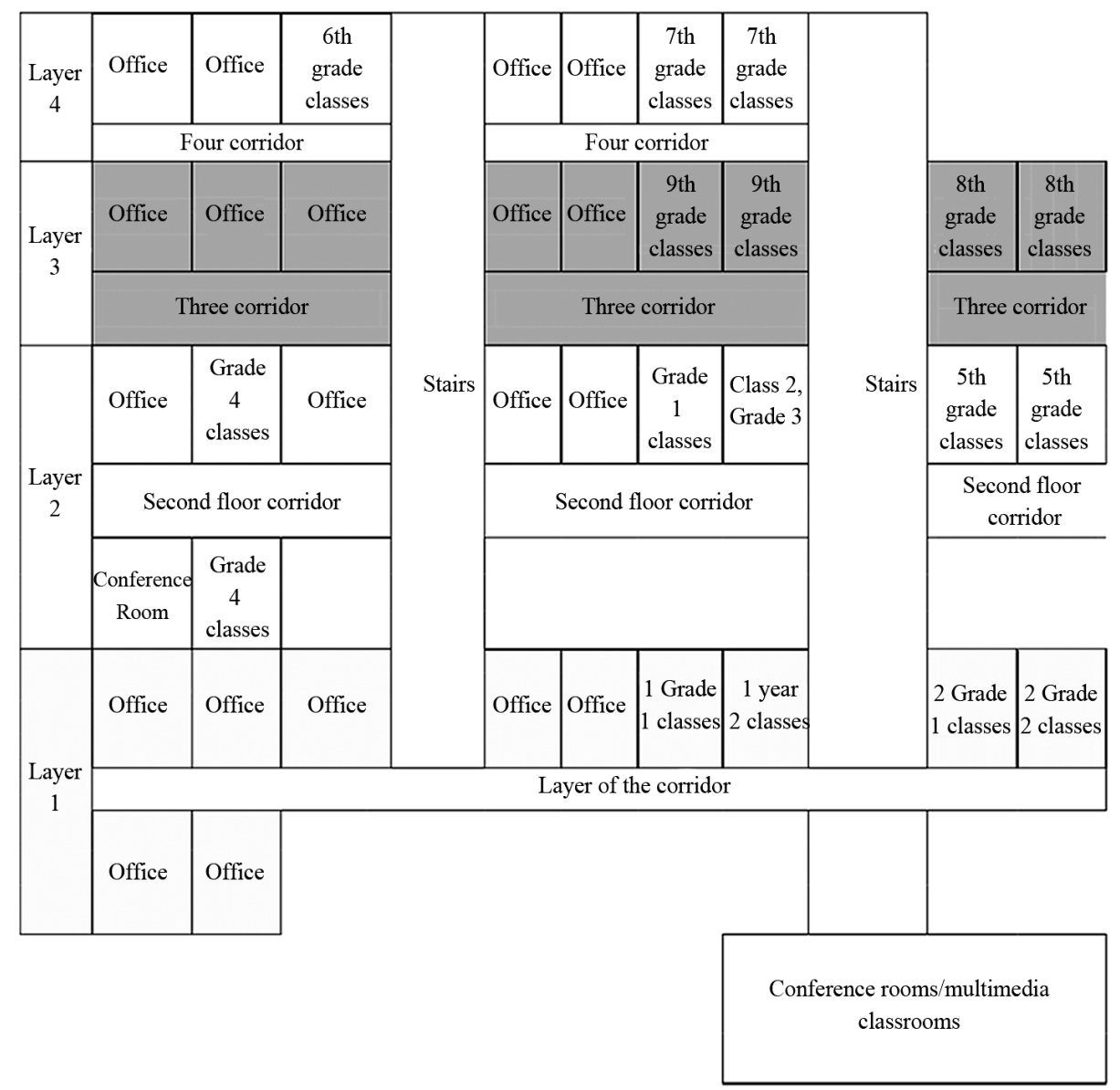

Figure 1. Floor plan of the teaching building. 
(64.29\%). Some cases also experienced a runny nose, nasal congestion, sneezing, nausea, vomiting, and diarrhea. Only one case developed pneumonia (Figure 2).

\subsubsection{Time Distribution}

The first case developed on March 17th. The number of cases started to rise from the March 19th until April 1st; a total of 40 people developed the disease (Figure 3).

\subsubsection{Population Distribution}

\section{1) Occupation}

There were 38 student infections (attack rate 5.98\%) and two infections among teachers (attack rate $2.78 \%$ ).

\section{2) Gender}

There were 25 male cases and 15 female cases, with a male to female ratio of $1.67: 1$.

\section{3) Age}

Most of the cases were aged 13 years old $(n=14)$ and 14 years old $(n=18)$, accounting for $35.0 \%$ and $45.0 \%$ of the total incidence. Five cases were aged 15, accounting for $12.5 \%$ of the total incidence.

\section{4) Class distribution}

The reported 38 student cases were mainly from class 1 and class 2 in grade 8 . The attack rates of the two classes were $52.78 \%$ for class 1 and $36.36 \%$ for class 2 . Compared to the total attack rate, the relative risk for these two classes were 2.57 and 2.21 respectively. Class 2 in grade 7 had the third highest attack rate, where four students were infected. The attack rate for this class was $15.4 \%$; other classes had below-average incidence rates (Table 1).

\subsubsection{Index Case Investigation}

The index case was a 14 year-old female student from class 1 in grade 8 . On the night of March 17th, she began coughing. Acute bronchitis syrup was self-administered but without relief. The patient attended class the following day while still coughing. On March 20th, she sought medical assistance in the Dingzhuang outpatient clinic and a five-day dose of anti-infection medication was prescribed. On March $25^{\text {th }}$, she developed a fever with a body temperature of $38.7^{\circ} \mathrm{C}$. On the same day, she was referred to the General Military Hospital. Azithromycin and Cofetol cough syrup were prescribed, but the symptoms persisted.

The patient was admitted to the Affiliated Hospital of Shandong University of Traditional Chinese Medicine on the morning of March 29th and underwent a CT scan. The results suggested lower-right inflammation of the lung. On the same afternoon the patient was referred back to the
Table 1. The grade distribution of 38 student cases.

\begin{tabular}{cccccc}
\hline Class & $\begin{array}{c}\text { Class } \\
\text { size }\end{array}$ & $\begin{array}{c}\text { Number } \\
\text { of cases }\end{array}$ & $\begin{array}{c}\text { Incidence } \\
\text { rate (\%) }\end{array}$ & RR & $95 \% \mathrm{CI}$ \\
\hline Class 1 grade 8 & 36 & 19 & 52.78 & 2.57 & $1.69-3.91$ \\
Class 2 grade 8 & 33 & 12 & 36.36 & 2.21 & $0.93-5.23$ \\
Class 2 grade 7 & 26 & 4 & 15.38 & 0.75 & $0.29-1.93$ \\
Class 1 grade 6 & 54 & 2 & 3.70 & 0.18 & $0.04-0.72$ \\
Class 2 grade 3 & 36 & 1 & 2.78 & 0.14 & $0.02-0.95$ \\
Total & 185 & 38 & 20.54 & REF & \\
\hline
\end{tabular}

Note: two cases of teachers were not included in this analysis.

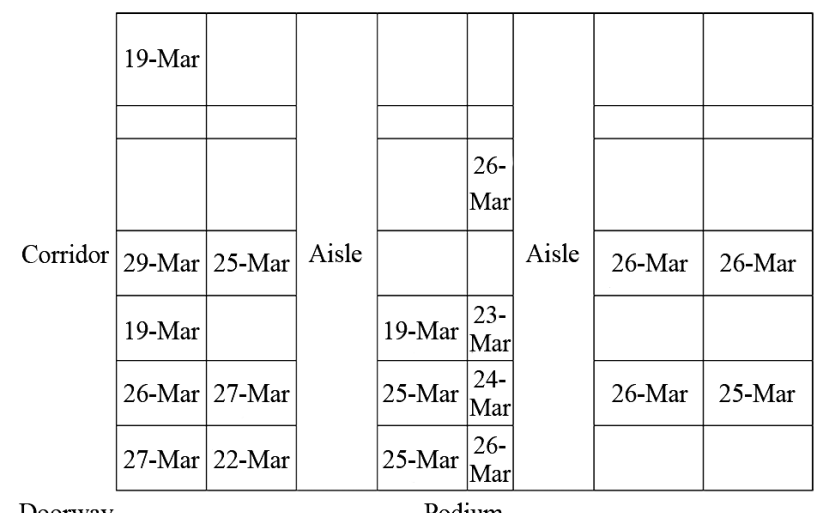

Doorway

Figure 2. Main symptoms distribution of cases.

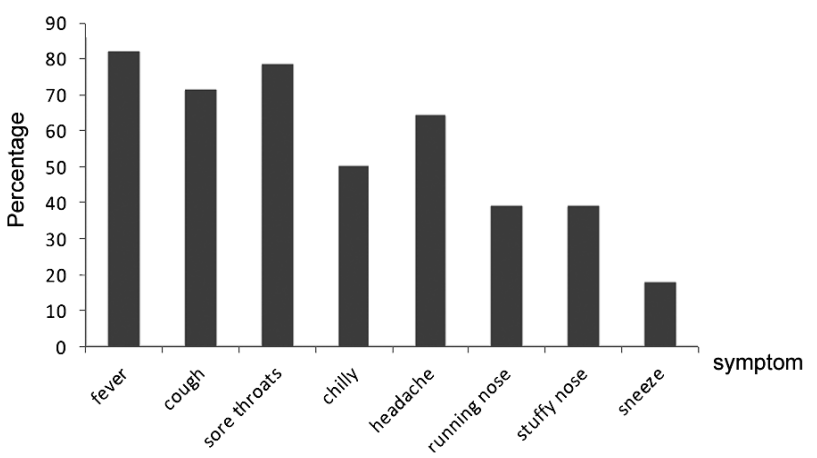

Figure 3. Cases onset time distribution.

Jinan Military Region General Hospital and was hospitalized. During the hospitalization period fever returned with a body temperature of $39.9^{\circ} \mathrm{C}$.

\section{1) Laboratory Diagnosis}

A routine blood examination was carried out on March 30th. The results showed: white blood cell (WBC) counts of $13.22 \times 10^{9}$, mononuclear cells $11.3 \%$, a weak positive test for mycoplasma pneumoniae serology, sputum culture growth of Streptococcus viridans, cardiac enzymes (creatine kinase isoenzymes 30, myocardial ischemia modified albumin 79.6, creatine kinase 374 , $\alpha$-hydroxy- 
butyrate the dehydrogenation acid 200). Another routine blood examination was carried out on April 1st on the index case, and the results showed that the WBC count had dropped to $6.34 \times 10^{9}$ and the mononuclear count was $11.2 \%$.

\section{2) Hospitalization}

Treatment administered to the index case during hospitalization focused on anti-inflammation and symptom relief. Medicines prescribed included $2.0 \mathrm{~g}$ ceftriaxone, 60 $\mathrm{mg}$ ambroxol hydrochloride intravenous infusion (once a day). On March 31st the body temperature of the patient began to fall and most symptoms improved; however, in the morning diarrhea occurred, which lasted for one day with a frequency of 4 - 5 times/day. By April 3rd, the patient was fully recovered and discharged from the hospital.

\subsubsection{Key Case Investigation}

The key case was a 35-year-old male teacher. He was the Chinese teacher of the 8th grade as well as the class adviser for class 2 in grade 8 . He was responsible for five lessons per class in grade 8 per week. On March 26th, he started to develop fever (body temperature of $39.5^{\circ} \mathrm{C}$ ) as well as body aches and tonsillar inflammation. He asked for sick leave on March 27th and was given cephalosporins and levofloxacin at a local clinic. The next day he returned to school as his body temperature had dropped. On March 29th, however, he had a recurrent fever and headache; azithromycin and ribavirin were prescribed by the clinic until the symptoms completely resolved.

The key case quarantined himself at home on the first day that he started to develop symptoms (March 26th); he also wore a mask and dined separately. His wife experienced an upper respiratory tract infection on the April $1^{\text {st; }}$ other family members did not experience any symptoms.

\section{Etiological Examinations}

\subsection{RT-PCR}

Multiplex PCR was used to examine 12 of the 21 throat swabs. The results were negative for influenza type A and type $\mathrm{B}$ virus, respiratory syncytial virus, rhinovirus, parainfluenza virus, coronavirus, and bocavirus nucleic acid. All 21 throat swab specimens were tested for AdV nucleic acid and 18 were found positive (positive rate of $85.71 \%)$.

\subsection{Virus Isolation}

All 21 throat swabs were isolated and cultured with Hep 2 and Vero cells. Two specimens were cytopathic $(9.52 \%)$, and PCR showed the presence of AdV.

\subsection{AdV HEXON Gene Phylogenetic Analysis}

Three positive specimens were sequenced for HEXON and were named 2012-JN-357, 2012-JN-358, and 2012$\mathrm{JN}-362$. The total length was 2841 bases. They were then analyzed for nucleotide acid sequence homology with MegAlign. Sequencing alignment was carried out and a phylogenetic tree was built using Muscle of Mega5 (Figure 4). The three sequences in the gene evolutionary tree located on the 55 branches of the AdV group B, were all shown to have $100 \%$ homology with the AdV 55-type control strain (GenBank Accession No. DQ874353.2).

\subsection{Disseminating Factor Analysis}

\subsubsection{Time Analysis}

The first infection occurred on March 17th, and the patient attended school until March 25th. The first generation of cases $(n=4)$ developed from March 17th - 22nd, which were all from class 1 in grade 8 . The second generation of cases $(n=36)$ developed from March 25th to April $1^{\text {st. }}: 15$ from class 1 in grade 8,12 from class 2 in grade 8,4 from class 2 in grade 7, 2 from class 1 in grade 6,1 from class 2 in grade 3 , and two teachers.

\subsubsection{Classrooms Location Analysis}

- Cases from class 1 in grade 8 were mostly sitting near the first case.

- Class 2 in grade 8 was located next to class 1 in grade 8. The same teacher was in charge of both classes, and he was considered the source of infection.

- Both class 2 in grade 3 and class 2 in grade 7 were located on the east wing of the building. Cases from these two classes appeared during the late phase of the outbreak. It is suspected that the shared stairs was the route of transmission. Even though class 1 in grade 6 was located on the west wing of the building, all cases from this class were infected on March 31st. We suggest that they were infected by the already spread outbreak in the school (Figures $\mathbf{1}$ and 5).

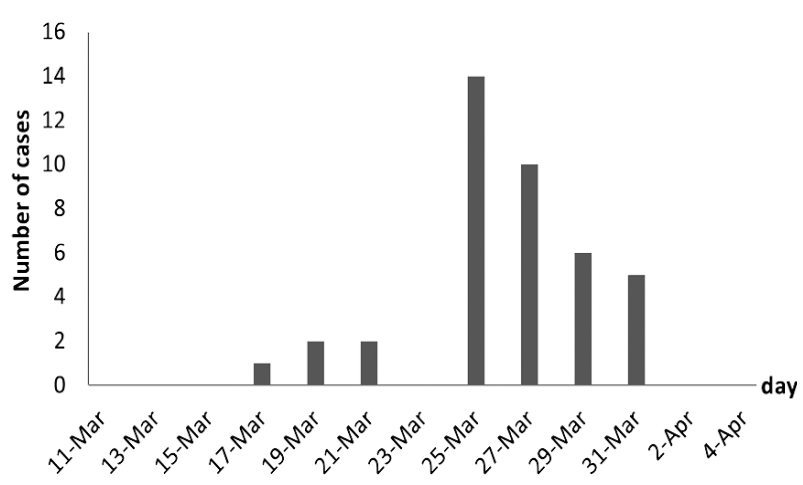

Figure 4. Complete HEXON gene sequence and AdV reference sequence gene phylogenetic tree. 


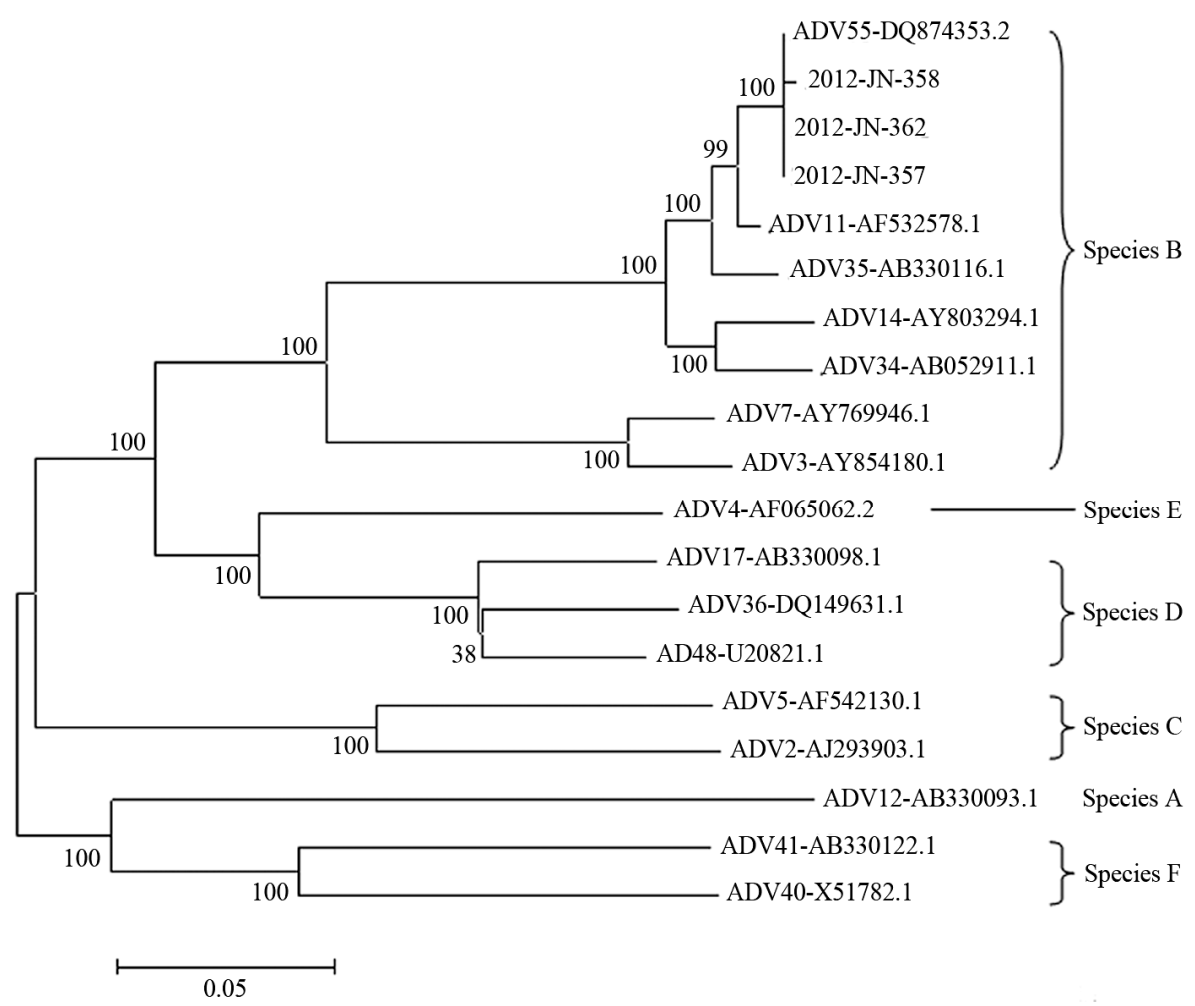

Figure 5. Eighth grade classes onset student seating distribution.

\subsection{On-Site Control Measures}

All reported cases were quarantined for observation and treatment. Since a large number of patients were identified in a short period of time, it was recommended to the school that it should have a seven-day holiday, combined with the Ching Ming Festival public holiday. The advisor teacher of each class should register any new incidence and report to the Jinan municipal center for disease controll and prevention in a timely manner. Classrooms should be thoroughly ventilated before school starts again and morning checks should be implemented. Educational flyers and notice boards should be used regarding the outbreak as well as to eliminate unnecessary panic.

\section{Discussion}

Based on the clinical manifestations of reported cases in combination with laboratory examination results, this outbreak was identified as an AdV-induced acute respiratory disease outbreak. AdV infections mostly occur in the winter and spring, and often cause outbreaks in the military [6-9]. As the disease usually manifests a series of common symptoms such as fever, sore throat, and coughing; this presented difficulties in determining the cause of the outbreak at an early stage [10]. With the evidence provided by timely and accurate laboratory diagnosis, the outbreak was able to be identified within the shortest time possible.

Respiratory infectious diseases normally spread rapidly and involve a large number of people. The transmission route is normally complex and the first case is often difficult to identify. In the present study, after carrying out a field investigation of the outbreak, it was determined that the first case was a 14 year-old female student. Since students from different classes are relatively isolated, we suggest the mutual teacher who was also infected spread the disease across classes. Effective morning checks and registration of student absences are important measures for preventing the spread of the disease within a school.

Human AdV can be divided into A $\sim \mathrm{G} 7$ groups and 57 types based on their physical, chemical, and biological properties. AdV infections induced by type 1, 2, 3, 5, and $6 \mathrm{AdV}$ are more commonly seen, and the main symptoms include fever, coughing, sore throat, and pneumonia; conjunctivitis is seen in some cases [11]. AdV is one of the main causes of acute respiratory infections among children in China. Child acute laryngitis is mostly induced by type $1,2,3$, and 5 human AdV, and child atypical pneumonia is mostly induced by type $1,3,4$, and 7 human AdV. Pathogens for conjunctivitis are usually type 3, 7, and 14 human AdV; epidemic keratoconjunctivitis is mostly caused by type 8 human AdV. Reports of AdVinduced infant acute diarrhea have also been seen $[12,13]$. In recent years, type $55 \mathrm{AdV}$ has been responsible for 
multiple outbreaks in the military and in school. Due to the lack of laboratory support in the past, many of the outbreaks have been misidentified as influenza epidemics [14]. With increasing use of nucleic acid testing technologies, the ability to correctly recognise infectious pathogens has been substantially improved. Viruses tend to survive longer in the winter and spring due to the low temperature. In addition, students tend to conduct more indoor activities during the winter and early spring, in a relatively closed space with poor ventilation. Both factors contribute to viral respiratory disease transmission. Consequently, winter and spring are peak seasons for type $55 \mathrm{AdV}$ infection to occur.

\section{Conclusion}

The outbreak originated from an adenovirus-infected student, who spread the pathogen to her classmates and teacher. The teacher then further disseminated the disease within the school which led to 40 febrile respiratory infections.

\section{Acknowledgements}

Thanks to Dr. Ji Zhang, for linguistic advice, Jinan Municipal Center for Disease Control \& Prevention.

\section{REFERENCES}

[1] J. P. Lynch, M. Fishbein and M. Echavarria, "Adenovirus. Seminars in Respiratory and Critical Care Medicine," Thieme Medical Publishers, 2011.

[2] M. Echavarria, et al., "Rapid Detection of Adenovirus in Throat Swab Specimens by PCR during Respiratory Disease Outbreaks among Military Recruits," Journal of Clinical Microbiology, Vol. 41, No. 2, 2003, pp. 810-812. http://dx.doi.org/10.1128/JCM.41.2.810-812.2003

[3] P. Yu, et al., "An Outbreak of Acute Respiratory Disease Caused by 'Human Adenovirus Type 7' in a Military Training Camp in Shaanxi, China. Microbiology and Immunology," 2013. http://dx.doi.org/10.1111/1348-0421.12074

[4] Z. Yang, et al., "Genomic Analyses of Recombinant Adenovirus Type 11a in China," Journal of Clinical Microbi- ology, Vol. 47, No. 10, 2009, pp. 3082-3090. http://dx.doi.org/10.1128/JCM.00282-09

[5] M. P. Walsh, et al., "Computational Analysis Identifies Human Adenovirus Type 55 as a Re-Emergent Acute Respiratory Disease Pathogen," Journal of Clinical Microbiology, Vol. 48, No. 3, 2010, pp. 991-993. http://dx.doi.org/10.1128/JCM.01694-09

[6] S. Dey, et al., "Short Report Prevalence, Seasonality, and Peak Age of Infection of Enteric Adenoviruses in Japan, 1995-2009," 2012.

[7] L. Zou, et al., "Human Adenovirus Infection in Children with Acute Respiratory Tract Disease in Guangzhou, China," Apmis, Vol. 120, No. 8, 2012, pp. 683-688. http://dx.doi.org/10.1111/j.1600-0463.2012.02890.x

[8] A. Hong-Wu, et al., "Research on Etiology of Acute Respiratory Tract Infection in Children from Wuhan during Winter and Spring," Chinese Journal of Nosocomiology, Vol. 5, 2012, p. 095.

[9] S. Fletcher, et al., "Gastrointestinal Pathogen Distribution in Symptomatic Children in Sydney, Australia," Journal of Epidemiology and Global Health, 2013.

[10] T. V. Chan, "The Patient with Sore Throat," Medical Clinics of North America, Vol. 94, No. 5, 2010, p. 923. http://dx.doi.org/10.1016/j.mcna.2010.06.001

[11] T. C. Mast, et al., "International Epidemiology of Human Pre-Existing Adenovirus (Ad) Type-5, Type-6, Type-26 and Type-36 Neutralizing Antibodies: Correlates of High Ad5 Titers and Implications for Potential HIV Vaccine Trials," Vaccine, Vol. 28, No. 4, 2010, pp. 950-957. http://dx.doi.org/10.1016/j.vaccine.2009.10.145

[12] G. Cleghorn, et al., "Acute Infectious Diarrhea Lessons Learned from the Past?" Journal of Pediatric Gastroenterology and Nutrition, Vol. 55, No. 5, 2012, pp. 489-493. http://dx.doi.org/10.1097/MPG.0b013e318272acc6

[13] B. Lan, et al., "Analysis on Epidemiologic Characteristics of Viral Diarrhea among Infants in Taiyuan, Shanxi Province, 2007-2008]," Chinese Journal of Experimental and Clinical Virology, Vol. 24, No. 1, 2010, p. 8.

[14] L. van der Hoek, et al., "Identification of a New Human Coronavirus," Nature Medicine, Vol. 10, No. 4, 2004, pp. 368-373. http://dx.doi.org/10.1038/nm1024 